\title{
WEIGHTED INFINITESIMAL UNITARY BIALGEBRAS, PRE-LIE, MATRIX ALGEBRAS AND POLYNOMIAL ALGEBRAS
}

\author{
YI ZHANG, JIA-WEN ZHENG, AND YAN-FENG LUO
}

\begin{abstract}
AвSTRACT. Motivated by comatrix coalgebras, we introduce the concept of a Newtonian comatrix coalgebra. We construct an infinitesimal unitary bialgebra on matrix algebras, via the construction of a suitable coproduct. As a consequence, a Newtonian comatrix coalgebra is established. Furthermore, an infinitesimal unitary Hopf algebra, under the view of Aguiar, is constructed on matrix algebras. By the close relationship between pre-Lie algebras and infinitesimal unitary bialgebras, we erect a pre-Lie algebra and a new Lie algebra on matrix algebras. Finally, a weighted infinitesimal unitary bialgebra on non-commutative polynomial algebras is also given.
\end{abstract}

\section{Contents}

1. Introduction 1

2. Weighted infinitesimal unitary bialgebras and infinitesimal unitary Hopf algebras 3

2.1. Weighted infinitesimal unitary bialgebras 3

2.2. Infinitesimal unitary Hopf algebras under the view of Aguiar 5

3. Infinitesimal unitary Hopf algebras and pre-Lie algebras on matrix algebras 6

$\begin{array}{lll}\text { 3.1. An infinitesimal unitary bialgebra on matrix algebras } & 7\end{array}$

3.2. An infinitesimal unitary Hopf algebra on matrix algebras 10

3.3. A pre-Lie and a new Lie algebraic structures on matrix algebras 11

4. Weighted infinitesimal unitary bialgebras on polynomial algebras 13

$\begin{array}{ll}\text { References } & 17\end{array}$

\section{INTRODUCTION}

A weighted infinitesimal unitary bialgebra is a module $A$ which is simultaneously an algebra (possibly without a unit) and a coalgebra (possibly without a counit) such that the coproduct $\Delta$ is a weighted derivation of $A$ in the sense that

$$
\Delta(a b)=a \cdot \Delta(b)+\Delta(a) \cdot b+\lambda(a \otimes b) \text { for } a, b \in A,
$$

where $\lambda \in \mathbf{k}$ is a fixed constant.

Weighted infinitesimal unitary bialgebras, first appeared in [35] and further studied in [12, 23, 43], are in order to give an algebraic meaning of non-homogenous associative classical YangBaxter equations in the context of associative algebras [35]. It should be pointed out that the weighted infinitesimal unitary bialgebra is a uniform version of two infinitesimal bialgebras. The first version of infinitesimal bialgebras, also called Newtonian coalgebra [27], introduced by Joni and Rota [28], are aimed at giving an algebraic framework for the calculus of Newton divided

Date: February 28, 2022.

2010 Mathematics Subject Classification. 15A30, 16W99, 16T10, 17B60, 17D25 .

Key words and phrases. matrix algebra, polynomial algebra, infinitesimal bialgebra, pre-Lie algebra. 
differences. Namely, an infinitesimal bialgebra is a module $A$ which is simultaneously an algebra (possibly without a unit) and a coalgebra (possibly without a counit) such that the coproduct $\Delta$ is a derivation of $A$ in the sense that

$$
\Delta(a b)=a \cdot \Delta(b)+\Delta(a) \cdot b \text { for } a, b \in A .
$$

The basic theory of infinitesimal bialgebras and infinitesimal Hopf algebras was developed by Aguiar [1, 3, 4, 5], has proven useful not only in combinatorices [4, 18], but in other areas of mathematics as well, such as associative Yang-Baxter equations [1, 5], Drinfeld's doubles [1, 39] and pre-Lie algebras [1]. The second version of infinitesimal bialgebras was defined by Loday and Ronco [31] and brought new life on rooted trees by Foissy [21, 22] in the sense that

$$
\Delta(a b)=a \cdot \Delta(b)+\Delta(a) \cdot b-a \otimes b \text { for } a, b \in A .
$$

In 2010, Ogievetsky and Popov [35] showed that weighted infinitesimal unitary bialgebras play an important role in mathematical physics. Given a solution $r \in A \otimes A$ of the non-homogenous associative classical Yang-Baxter equation, one can construct a weighted infinitesimal unitary bialgebra [35], involving a coproduct given by

$$
\Delta_{r}(a):=a \cdot r-r \cdot a-\lambda(a \otimes 1) \text { for } a \in A .
$$

Weighted infinitesimal unitary bialgebras also have found applications in combinatorics. Based on Hochschild 1-cocycle conditions in Cartier-Quillen cohomology, we can construct weighted infinitesimal unitary bialgebras on various combinatorial objects, such as planar rooted forests and decorated rooted forests [21, 23, 40, 42]. A surprising phenomena showed that these weighted infinitesimal unitary bialgebras can be treated in the framework of operated algebras, via grafting operators $[40,42]$. It has been observed that the weighted infinitesimal unitary bialgebras on rooted forests possess universal properties. In particular, the objects studied in the wellknown Connes-Kreimer Hopf algebra have a free cocycle weighted infinitesimal bialgebraic structure [23, 40, 42]. Thus it would be interesting to construct a number of weighted infinitesimal bialgebras on some combinatorial objects or various well-known algebras. This is our main goal of this paper.

Let $M_{n}(\mathbf{k})$ be the matrix algebra and $\left\{E_{i j}\right\}_{1 \leq i, j \leq n}$ the canonical k-basis for $M_{n}(\mathbf{k})$. Then $M_{n}(\mathbf{k})$ has a coalgebraic structure [37] determined by

$$
\Delta\left(E_{i j}\right):=\sum_{s=1}^{n} E_{i s} \otimes E_{s j} \text { and } \varepsilon\left(E_{i j}\right):=\delta_{i j},
$$

where $\delta_{i j}$ is the Kronecker function. This comatrix coalgebra plays a foundmental role in classical bialgebras. It is almost a natural question to wonder whether we can construct an infinitesimal bialgebra on $M_{n}(\mathbf{k})$. This paper gives a positive answer to this question. Strongly motivated by the construction of comatrix coalgebra, we show that $M_{n}(\mathbf{k})$ possesses an infinitesimal unitary bialgebraic structure with the coproduct defined by

$$
\Delta_{\epsilon}\left(E_{i j}\right):= \begin{cases}\sum_{s=i}^{j-1} E_{i s} \otimes E_{(s+1) j} & \text { if } i<j, \\ 0 & \text { if } i=j, \\ -\sum_{s=j}^{i-1} E_{i s} \otimes E_{(s+1) j} & \text { if } i>j .\end{cases}
$$

We call this infinitesimal unitary bialgebra a Newtonian comatrix coalgebra. We emphasize that the Newtonian comatrix coalgebra is different from the one introduced in [43]. See Remark 3.5 below. Moreover, we equip an infinitesimal unitary bialgebra on matrix algebras with an antipode such that it is further an infinitesimal unitary Hopf algebra, under the view of Aguiar [1]. As a 
related result, an infinitesimal unitary bialgebra of weight $\lambda$ on non-commutative polynomial algebras is also given.

Pre-Lie algebras, also called Vinberg algebras, first appeared in the work of Vinberg [38] under the name left-symmetric algebras on cones and also appeared independently in the study of deformation and cohomology of associative algebras [24]. Later, there have been several interesting developments of pre-Lie algebras in mathematices and mathematical physics, such as classical and quantum Yang-Baxter equations [9, 13, 19, 26], Lie groups and Lie algebras [7, 29, 34, 38], prePoisson algebras [2] and Poisson brackets [14], quantum field theory [15] and operads [16, 36], $\mathcal{O}$-operators [8, 10] and Rota-Baxter algebras [6, 11, 25, 32]. Because of the non-associativity of pre-Lie algebras, there is not a suitable representation theory and not a complete structure theory of pre-Lie algebras [8]. It is natural to consider how to construct them from some algebraic structures which we have known. The present paper is an attempt to construct pre-Lie algebraic structures on some associative algebras, especially on matrix algebras. In the algebraic framework of Aguiar [5] for infinitesimal bialgebras, a pre-Lie algebraic structure is constructed from an arbitrary infinitesimal (unitary) bialgebra. As an application, a pre-Lie algebraic structure and then a new Lie algebraic structure on matrix algebras are built in this paper.

Notation. Throughout this paper, let $\mathbf{k}$ be a unitary commutative ring unless the contrary is specified, which will be the base ring of all modules, algebras, coalgebras, bialgebras, tensor products, as well as linear maps. By an algebra we mean an associative algebra (possibly without a unit) and by a coalgebra we mean a coassociative coalgebra (possibly without a counit). For an algebra $A$, we view $A \otimes A$ as an $(A, A)$-bimodule via

$$
a \cdot(b \otimes c):=a b \otimes c \text { and }(b \otimes c) \cdot a:=b \otimes c a,
$$

where $a, b, c \in A$. We shall use the sign function $\operatorname{sgn}(x), x \in Z$, which is given by

$$
\operatorname{sgn}(x):= \begin{cases}1 & \text { if } x>0 \\ 0 & \text { if } x=0 \\ -1 & \text { if } x<0\end{cases}
$$

\section{WEIGHTED INFINITESIMAL UNITARY BIALGEBRAS AND INFINITESIMAL UNITARY HoPF ALGEBRAS}

In this section, we first recall the concept of a weighted infinitesimal (unitary) bialgebra [23], which generalise simultaneously the one introduced by Joni and Rota [28] and the one initiated by Loday and Ronco [31]. We then recall the notation of an infinitesimal (unitary) Hopf algebra, under the view of Aguiar.

2.1. Weighted infinitesimal unitary bialgebras. The following is the concept of a weighted infinitesimal (unitary) bialgebra proposed in [23].

Definition 2.1. [23] Let $\lambda$ be a given element of $\mathbf{k}$. An infinitesimal bialgebra (abbreviated $\epsilon$-bialgebra) of weight $\lambda$ is a triple $(A, m, \Delta)$ consisting of an algebra $(A, m)$ (possibly without a unit) and a coalgebra $(A, \Delta)$ (possibly without a counit) that satisfies

$$
\Delta(a b)=a \cdot \Delta(b)+\Delta(a) \cdot b+\lambda(a \otimes b), \quad \forall a, b \in A .
$$

If further $(A, m, 1)$ is a unitary algebra, then the quadruple $(A, m, 1, \Delta)$ is called an infinitesimal unitary bialgebra (abbreviated $\epsilon$-unitary bialgebra) of weight $\lambda$.

The concept of an $\epsilon$-bialgebra morphism is given as usual. 
Definition 2.2. [23] Let $A$ and $B$ be two $\epsilon$-bialgebras of weight $\lambda$. A map $\phi: A \rightarrow B$ is called an infinitesimal bialgebra morphism (abbreviated $\epsilon$-bialgebra morphism) if $\phi$ is an algebra morphism and a coalgebra morphism. The concept of an infinitesimal unitary bialgebra morphism can be defined in the same way.

Remark 2.3. (a) The $\epsilon$-bialgebra introduced by Joni and Rota [28] is of weight zero and the $\epsilon$-bialgebra originated from Loday and Ronco [31] is of weight -1 .

(b) Let $(A, m, 1, \Delta)$ be an $\epsilon$-unitary bialgebra of weight $\lambda$. Then $\Delta(1)=-\lambda(1 \otimes 1)$ by

$$
\Delta(1)=\Delta(1 \cdot 1)=1 \cdot \Delta(1)+\Delta(1) \cdot 1+\lambda(1 \otimes 1)=2 \Delta(1)+\lambda(1 \otimes 1) .
$$

(c) Aguiar [1] pointed out that there is no nonzero $\epsilon$-bialgebra of weight zero which is both unitary and counitary. Indeed, it follows the counicity that

$$
1 \otimes 1_{\mathbf{k}}=(\mathrm{id} \otimes \varepsilon) \Delta(1)=0
$$

and so $1=0$.

Example 2.4. Here are some examples of weighted $\epsilon$-(unitary) bialgebras.

(a) Any unitary algebra $\left(A, m, 1_{A}\right)$ is an $\epsilon$-unitary bialgebra of weight zero when the coproduct is taken to be $\Delta=0$.

(b) [1, Example 2.3.5] The polynomial algebra $\mathbf{k}\left\langle x_{1}, x_{2}, x_{3}, \ldots\right\rangle$ is an $\epsilon$-unitary bialgebra of weight zero with the coproduct $\Delta$ given by Eq. (2) and

$$
\Delta\left(x_{n}\right)=\sum_{i=0}^{n-1} x_{i} \otimes x_{n-1-i}=1 \otimes x_{n-1}+x_{1} \otimes x_{n-2}+\cdots+x_{n-1} \otimes 1,
$$

where we set $x_{0}=1$.

(c) [1, Example 2.3.2] Let $Q$ be a quiver. The path algebra of $Q$ is the associative algebra $\mathbf{k} Q=\oplus_{n=0}^{\infty} \mathbf{k} Q_{n}$ whose underlying module has its basis the set of all paths $a_{1} a_{2} \cdots a_{n}$ of length $n \geq 0$ in $Q$. The multiplication $*$ of two paths $a_{1} a_{2} \cdots a_{n}$ and $b_{1} b_{2} \cdots b_{m}$ is defined by

$$
\left(a_{1} a_{2} \cdots a_{n}\right) *\left(b_{1} b_{2} \cdots b_{m}\right):=\delta_{t\left(a_{n}\right), s\left(b_{1}\right)} a_{1} a_{2} \cdots a_{n} b_{1} b_{2} \cdots b_{m},
$$

where $\delta_{t\left(a_{n}\right), s\left(b_{1}\right)}$ is the Kronecker delta. The path algebra $\left(\mathbf{k} Q, *, \Delta_{\epsilon}\right)$ is an $\epsilon$-bialgebra of weight zero with the coproduct defined by

$$
\begin{aligned}
& \Delta(e):=0 \text { for } e \in Q_{0} \\
& \Delta(a):=s(a) \otimes t(a) \text { for } a \in Q_{1}, \text { and }
\end{aligned}
$$

$\Delta\left(a_{1} a_{2} \cdots a_{n}\right):=s\left(a_{1}\right) \otimes a_{2} \cdots a_{n}+a_{1} \cdots a_{n-1} \otimes t\left(a_{n}\right)+\sum_{i=1}^{n-2} a_{1} \cdots a_{i} \otimes a_{i+2} \cdots a_{n}$ for $n \geq 2$.

(d) [31, Section 2.3] Let $V$ denote a vector space. Recall that the tensor algebra $T(V)$ over $V$ is the tensor module,

$$
T(V)=\mathbf{k} \oplus V \oplus V^{\otimes 2} \oplus \cdots \oplus V^{\otimes n} \oplus \cdots,
$$

equipped with the associative multiplication $m_{T}$ called concatenation defined by

$$
v_{1} \cdots v_{i} \otimes v_{i+1} \cdots v_{n} \mapsto v_{1} \cdots v_{i} v_{i+1} \cdots v_{n} \text { for } 0 \leq i \leq n,
$$


WEIGHTED INFINITESIMAL UNITARY BIALGEBRAS, MATRIX ALGEBRAS AND POLYNOMIAL ALGEBRAS 5

and with the convention that $v_{1} v_{0}=1$ and $v_{n+1} v_{n}=1$. The tensor algebra $T(V)$ is an $\epsilon$-unitary bialgebra of weight -1 with the coassociative coproduct defined by

$$
\Delta\left(v_{1} \cdots v_{n}\right):=\sum_{i=0}^{n} v_{1} \cdots v_{i} \otimes v_{i+1} \cdots v_{n} .
$$

(e) [41, Example 2.4] The polynomial algebra $\mathbf{k}[x]$ is an $\epsilon$-unitary bialgebra of weight $\lambda$ with the coproduct defined by

$$
\Delta(1):=-\lambda(1 \otimes 1) \text { and } \Delta\left(x^{n}\right):=\sum_{i=0}^{n-1} x^{i} \otimes x^{n-1-i}+\lambda \sum_{i=1}^{n-1} x^{i} \otimes x^{n-i} \quad \text { for } n \geq 1 .
$$

When $\lambda=0$, this $\epsilon$-unitary bialgebra of weight zero is precisely the Newtonian coalgebra on $\mathbf{k}[x]$, which was constructed and studied by Hirschhorn and Raphael [27].

(f) [20, Section 1.4] Let $(A, m, 1, \Delta, \varepsilon, c)$ be a braided bialgebra with $A=\mathbf{k} \oplus \operatorname{ker} \varepsilon$ and the braiding $c: A \otimes A \rightarrow A \otimes A$ given by

$$
c:\left\{\begin{aligned}
1 \otimes 1 & \mapsto 1 \otimes 1, \\
a \otimes 1 & \mapsto \otimes a, \\
1 \otimes b & \mapsto b \otimes 1, \\
a \otimes b & \mapsto 0,
\end{aligned}\right.
$$

where $a, b \in \operatorname{ker} \varepsilon$ and $\lambda \in \mathbf{k}$. Then $(A, m, 1, \Delta)$ is an $\epsilon$-unitary bialgebra of weight -1 .

2.2. Infinitesimal unitary Hopf algebras under the view of Aguiar. Denote by $\operatorname{Hom}_{\mathbf{k}}(A, B)$ the set of linear maps from $A$ to $B$ throughout the remainder of this subsection.

Definition 2.5. [17, Chapter 4.1] Let $A=(A, m, 1, \Delta, \varepsilon)$ be a classical bialgebra. Then the convolution product $*$ on $\operatorname{Hom}_{\mathbf{k}}(A, A)$ defined to be the composition:

$$
f * g:=m(f \otimes g) \Delta \text { for } f, g \in \operatorname{Hom}_{\mathbf{k}}(A, A),
$$

and the triple $\left(\operatorname{Hom}_{\mathbf{k}}(A, A), *, 1 \circ \varepsilon\right)$ is called a convolution algebra, where $1 \circ \varepsilon$ is the unit with respect to $*$. The antipode $S$ is defined to be the inverse of the identity map with respect to the convolution product.

Remark 2.6. The question facing us is whether we can define the antipode for an $\epsilon$-unitary bialgebra of weight zero as one does for classical bialgebras. Aguiar [1, Remark 2.2] answered this question "No" in the case of weight zero due to the lack of the unit $1 \circ \varepsilon$ with respect to $*$, see Remark 2.3 (c).

To equip the $\epsilon$-bialgebra of weight zero with an antipode, Aguiar [1] introduced the notion of a circular convolution.

Definition 2.7. [1, Section 3] Let $(A, m, \Delta)$ be an $\epsilon$-bialgebra of weight zero. Then the circular convolution $\otimes$ on $\operatorname{Hom}_{\mathbf{k}}(A, A)$ defined by

$$
f \circledast g:=f * g+f+g, \text { that is, }(f \circledast g)(a):=\sum_{(a)} f\left(a_{(1)}\right) g\left(a_{(2)}\right)+f(a)+g(a) \text { for } a \in A .
$$

Note that $f \circledast 0=f=0 \circledast f$ and so $0 \in \operatorname{Hom}_{\mathbf{k}}(A, A)$ is the unit with respect to the circular convolution $\circledast$.

Further Aguiar [1] introduced the concept of an infinitesimal Hopf algebra via circular convolution. 
Definition 2.8. [1, Definition 3.1] An infinitesimal bialgebra $(A, m, \Delta)$ of weight zero is called an infinitesimal Hopf algebra (abbreviated $\epsilon$-Hopf algebra) if the identity map id $\in \operatorname{Hom}_{\mathbf{k}}(A, A)$ is invertible with respect to the circular convolution $\circledast$. In this case, the inverse $S \in \operatorname{Hom}_{\mathbf{k}}(A, A)$ of id is called the antipode of $A$. It is characterized by

$$
\sum_{(a)} S\left(a_{(1)}\right) a_{(2)}+S(a)+a=0=\sum_{(a)} a_{(1)} S\left(a_{(2)}\right)+a+S(a) \text { for } a \in A .
$$

Here $\Delta(a)=\sum_{(a)} a_{(1)} \otimes a_{(2)}$. If further $(A, m, 1, \Delta)$ is a unitary algebra, then $(A, m, 1, \Delta)$ is called an infinitesimal unitary Hopf algebra.

The $\epsilon$-unitary Hopf algebra satisfies many properties analogous to those of a classical Hopf algebra [1, Propositions 3.7, 3.12].

Remark 2.9. (a) Let $(A, m, \Delta)$ be an $\epsilon$-unitary Hopf algebra with antipode $S$. Then

$$
S(x y)=-S(x) S(y) \text { and } \sum_{(x)} S\left(x_{(1)}\right) \otimes S\left(x_{(2)}\right)=-\sum_{(S(x))} S(x)_{(1)} \otimes S(x)_{(2)}=-\Delta S(x) .
$$

(b) If $(A, m, \Delta)$ is an $\epsilon$-unitary Hopf algebra with antipode $S$, then so is $\left(A, m^{\mathrm{op}}, \Delta^{\mathrm{cop}}\right)$ with the same antipode $S$.

(c) It follows from Eq. (2.8) that $S(1)=-1$ by taking $a=1$.

Definition 2.10. [1, Section 4] Let $A$ be an algebra and $C$ a coalgebra. The map $f: C \rightarrow A$ is called locally nilpotent with respect to convolution $*$ if for each $c \in C$, there is some $n \geq 1$ such that

$$
f^{*(n)}(c):=\sum_{(c)} f\left(c_{(1)}\right) f\left(c_{(2)}\right) \cdots f\left(c_{(n+1)}\right)=0
$$

where $c_{(1)}, \cdots, c_{(n+1)}$ are from the Sweedler notation $\Delta^{n}(c)=\sum_{(c)} c_{(1)} \otimes \cdots \otimes c_{(n+1)}$ and $f^{* n}$ is defined inductively by

$$
f^{*(1)}(c):=\sum_{(c)} f\left(c_{(1)}\right) f\left(c_{(2)}\right) \text { and } f^{*(n)}:=f^{*(n-1)} * f .
$$

Denote by $\mathbb{R}$ and $\mathbb{C}$ the field of real numbers and the field of complex numbers, respectively.

Lemma 2.11. [1, Proposition 4.5] Let $(A, m, \Delta)$ be an $\epsilon$-bialgebra of weight zero and $D:=m \Delta$, and let $\mathbb{Q} \subseteq \mathbf{k}$. Suppose that either

(a) $\mathbf{k}=\mathbb{R}$ or $\mathbb{C}$ and $A$ is finite dimensional, or

(b) $D$ is locally nilpotent and $\operatorname{char}(\mathbf{k})=0$.

Then $A$ is an $\epsilon$-Hopf algebra with bijective antipode $S=-\sum_{n=0}^{\infty} \frac{1}{n !}(-D)^{n}$.

\section{INFINITESIMAL UNITARY HOPF ALGEBRAS AND PRE-LIE ALGEBRAS ON MATRIX ALGEBRAS}

In this section, we equip a matrix algebra $M_{n}(\mathbf{k})$ with an $\epsilon$-unitary Hopf algebraic structures, in terms of a construction of the suitable coproduct. In the algebraic framework of Aguiar [5] for $\epsilon$-(unitary) bialgebras, a pre-Lie and a new Lie algebraic structures on matrix algebras are constructed. 
3.1. An infinitesimal unitary bialgebra on matrix algebras. In this subsection, we construct an $\epsilon$-unitary bialgebra of weight zero arising from a matrix algebra.

Definition 3.1. [30, Chapter 17] Let $\mathbf{k}$ be a unitary commutative ring. A matrix algebra $M_{n}(\mathbf{k})$ is a collection of $n \times n$ matrices over $\mathbf{k}$ that form an associative algebra under matrix addition and matrix multiplication.

The multiplication on $M_{n}(\mathbf{k})$ will be denoted by $\mathrm{m}$. We now define a coproduct on matrix algebra $M_{n}(\mathbf{k})$ to equip it with a coalgebra structure, with an eye toward constructing an $\epsilon$-unitary bialgebra on it.

Let $E_{i j}, 1 \leq i, j \leq n$, be an elementary matrix whose entry in the $i$-th row, $j$-th column is 1 , and zero in all other entries. Note that $E_{i j} E_{k l}=\delta_{j k} E_{i l}$. Then any matrix $M \in M_{n}(\mathbf{k})$ can be decomposed as a linear combination of all the $n^{2}$ elementary matrices as follows:

$$
M=\left[m_{i j}\right]=\sum_{i, j=1}^{n} m_{i j} E_{i j}
$$

By linearity, we only need to define $\Delta_{\epsilon}\left(E_{i j}\right)$ for basis elements $E_{i j} \in M_{n}(\mathbf{k})$. Define

$$
\Delta_{\epsilon}\left(E_{i j}\right):= \begin{cases}\sum_{s=i}^{j-1} E_{i s} \otimes E_{(s+1) j} & \text { if } i<j, \\ 0 & \text { if } i=j, \\ -\sum_{s=j}^{i-1} E_{i s} \otimes E_{(s+1) j} & \text { if } i>j .\end{cases}
$$

We observe that $M_{n}(\mathbf{k})$ is closed under the coproduct $\Delta_{\epsilon}$.

Lemma 3.2. For $M, N \in M_{n}(\mathbf{k})$, we have

$$
\Delta_{\epsilon}(M N)=M \cdot \Delta_{\epsilon}(N)+\Delta_{\epsilon}(M) \cdot N .
$$

Proof. Let $E_{i j}$ and $E_{k l}$ be elementary matrices of $M, N$, respectively. Then it is enough to verify

$$
\Delta_{\epsilon}\left(E_{i j} E_{k l}\right)=E_{i j} \cdot \Delta_{\epsilon}\left(E_{k l}\right)+\Delta_{\epsilon}\left(E_{i j}\right) \cdot E_{k l} \text { for } 1 \leq i, j, k, l \leq n .
$$

We have two cases to consider.

Case 1. $j \neq k$. In this case, by Eq. (5), we have

$$
\Delta_{\epsilon}\left(E_{i j} E_{k l}\right)=\Delta_{\epsilon}(0)=0=E_{i j} \cdot \Delta_{\epsilon}\left(E_{k l}\right)+\Delta_{\epsilon}\left(E_{i j}\right) \cdot E_{k l}
$$

Case 2. $j=k, i \leq l$. In this case, we only need to consider the following three subcases.

Subcase 2.1. $i \leq j \leq l$. By Eq. (5), we have

$$
\begin{aligned}
E_{i j} \cdot \Delta_{\epsilon}\left(E_{k l}\right)+\Delta_{\epsilon}\left(E_{i j}\right) \cdot E_{k l} & =E_{i j} \cdot\left(\sum_{s=k}^{l-1} E_{k s} \otimes E_{(s+1) l}\right)+\left(\sum_{s=i}^{j-1} E_{i s} \otimes E_{(s+1) j}\right) \cdot E_{k l} \\
& =\sum_{s=j}^{l-1} E_{i s} \otimes E_{(s+1) l}+\sum_{s=i}^{j-1} E_{i s} \otimes E_{(s+1) l} \\
& =\sum_{s=i}^{l-1} E_{i s} \otimes E_{(s+1) l}=\Delta_{\epsilon}\left(E_{i l}\right)=\Delta_{\epsilon}\left(E_{i j} E_{k l}\right) .
\end{aligned}
$$

Subcase 2.2. $j<i \leq l$. By Eq. (5), we have

$$
E_{i j} \cdot \Delta_{\epsilon}\left(E_{k l}\right)+\Delta_{\epsilon}\left(E_{i j}\right) \cdot E_{k l}=E_{i j} \cdot\left(\sum_{s=k}^{l-1} E_{k s} \otimes E_{(s+1), l}\right)-\left(\sum_{s=j}^{i-1} E_{i s} \otimes E_{(s+1) j}\right) \cdot E_{k l}
$$




$$
\begin{aligned}
& =\sum_{s=j}^{l-1} E_{i s} \otimes E_{(s+1) l}-\sum_{s=j}^{i-1} E_{i s} \otimes E_{(s+1) l} \\
& =\sum_{s=i}^{l-1} E_{i s} \otimes E_{(s+1) l}=\Delta_{\epsilon}\left(E_{i l}\right)=\Delta_{\epsilon}\left(E_{i j} E_{k l}\right)
\end{aligned}
$$

Subcase 2.3. $i \leq l<j$. By Eq. (5), we have

$$
\begin{aligned}
E_{i j} \cdot \Delta_{\epsilon}\left(E_{k l}\right)+\Delta_{\epsilon}\left(E_{i j}\right) \cdot E_{k l} & =-E_{i j} \cdot \sum_{s=l}^{k-1} E_{k s} \otimes E_{(s+1) l}+\sum_{s=i}^{j-1} E_{i s} \otimes E_{(s+1) j} \cdot E_{k l} \\
& =-\sum_{s=l}^{j-1} E_{i s} \otimes E_{(s+1) l}+\sum_{s=i}^{j-1} E_{i s} \otimes E_{(s+1) l} \\
& =\sum_{s=i}^{l-1} E_{i s} \otimes E_{(s+1) l}=\Delta_{\epsilon}\left(E_{i l}\right)=\Delta_{\epsilon}\left(E_{i j} E_{k l}\right) .
\end{aligned}
$$

Case 3. $j=k, i>l$. It is similar to the proof of Case 2.

Lemma 3.3. The pair $\left(M_{n}(\mathbf{k}), \Delta_{\epsilon}\right)$ is a coalgebra (without counit).

Proof. It is enough to show the coassociative law:

$$
\left(\mathrm{id} \otimes \Delta_{\epsilon}\right) \Delta_{\epsilon}\left(E_{i j}\right)=\left(\Delta_{\epsilon} \otimes \mathrm{id}\right) \Delta_{\epsilon}\left(E_{i j}\right) \text { for } E_{i j} \in M_{n}(\mathbf{k}) .
$$

When $i=j$, the Eq. (6) holds trivially. By the definition of $\Delta_{\epsilon}$ in Eq. (5), we have two cases to consider.

Case 1. $i<j$. In this case, we have

$$
\begin{aligned}
\left(\mathrm{id} \otimes \Delta_{\epsilon}\right) \Delta_{\epsilon}\left(E_{i j}\right) & =\left(\mathrm{id} \otimes \Delta_{\epsilon}\right)\left(\sum_{s=i}^{j-1} E_{i s} \otimes E_{(s+1) j}\right) \quad(\text { by Eq. (5)) } \\
& =\sum_{s=i}^{j-1} E_{i s} \otimes \Delta_{\epsilon}\left(E_{(s+1) j}\right)=\sum_{s=i}^{j-2} E_{i s} \otimes \Delta_{\epsilon}\left(E_{(s+1) j}\right) \quad\left(\text { by } \Delta_{\epsilon}\left(E_{i i}\right)=0\right) \\
& =\sum_{s=i}^{j-2} \sum_{t=s+1}^{j-1} E_{i s} \otimes E_{(s+1) t} \otimes E_{(t+1) j}=\sum_{t=i+1}^{j-1} \sum_{s=i}^{t-1} E_{i s} \otimes E_{(s+1) t} \otimes E_{(t+1) j} \quad \text { (by Eq. (5)) } \\
& =\sum_{s=i+1}^{j-1} \sum_{t=i}^{s-1} E_{i t} \otimes E_{(t+1) s} \otimes E_{(s+1) j} \quad(\text { by exchanging the index } s \text { and } t) \\
& =\sum_{s=i+1}^{j-1} \Delta_{\epsilon}\left(E_{i s}\right) \otimes E_{(s+1) j}=\sum_{s=i}^{j-1} \Delta_{\epsilon}\left(E_{i s}\right) \otimes E_{(s+1) j} \quad\left(\text { by } \Delta_{\epsilon}\left(E_{i i}\right)=0\right) \\
& =\left(\Delta_{\epsilon} \otimes \mathrm{id}\right)\left(\sum_{s=i}^{j-1} E_{i s} \otimes E_{(s+1) j}\right)=\left(\Delta_{\epsilon} \otimes \mathrm{id}\right) \Delta_{\epsilon}\left(E_{i j}\right) \quad \text { (by Eq. (5)). }
\end{aligned}
$$

Case 2. $i>j$. In this case, we have

$\left(\mathrm{id} \otimes \Delta_{\epsilon}\right) \Delta_{\epsilon}\left(E_{i j}\right)=\left(\mathrm{id} \otimes \Delta_{\epsilon}\right)\left(-\sum_{s=j}^{i-1} E_{i s} \otimes E_{(s+1) j}\right) \quad$ (by Eq. (5)) 
WEIGHTED INFINITESIMAL UNITARY BIALGEBRAS, MATRIX ALGEBRAS AND POLYNOMIAL ALGEBRAS 9

$$
\begin{aligned}
& =-\sum_{s=j}^{i-1} E_{i s} \otimes \Delta_{\epsilon}\left(E_{(s+1) j}\right) \\
& =\sum_{s=j}^{i-1} \sum_{t=j}^{s} E_{i s} \otimes E_{(s+1) t} \otimes E_{(t+1) j}=\sum_{t=j}^{i-1} \sum_{s=t}^{i-1} E_{i s} \otimes E_{(s+1) t} \otimes E_{(t+1) j} \quad \text { (by Eq. (5)) } \\
& \left.=\sum_{s=j}^{i-1} \sum_{t=s}^{i-1} E_{i t} \otimes E_{(t+1) s} \otimes E_{(s+1) j} \quad \text { (by exchanging the index } s \text { and } t\right) \\
& =-\sum_{s=j}^{i-1} \Delta_{\epsilon}\left(E_{i s}\right) \otimes E_{(s+1) j}=-\left(\Delta_{\epsilon} \otimes \mathrm{id}\right)\left(\sum_{s=j}^{i-1} E_{i s} \otimes E_{(s+1) j}\right) \\
& =\left(\Delta_{\epsilon} \otimes \mathrm{id}\right) \Delta_{\epsilon}\left(E_{i j}\right) \quad(\text { by Eq. (5)). }
\end{aligned}
$$

This completes the proof.

Now we arrive at our main result in this subsection.

Theorem 3.4. The quadruple $\left(M_{n}(\mathbf{k}), \mathrm{m}, E, \Delta_{\epsilon}\right)$ is an $\epsilon$-unitary bialgebra of weight zero.

Proof. It follows from Lemmas 3.2 and 3.3.

Remark 3.5. (a) Let us emphasize that this $\epsilon$-unitary bialgebra on $M_{n}(\mathbf{k})$ is different from the one constructed in our previous paper [43]. Let $L \in M_{n}(\mathbf{k})$ such that $L^{2}=0$. Then the quadruple $\left(M_{n}(\mathbf{k}), \mathrm{m}, E, \Delta_{\epsilon}\right)$ is an $\epsilon$-unitary bialgebra of weight zero [43] with the coproduct defined by

$$
\Delta_{\epsilon}(M):=M L \otimes L-L \otimes L M \text { for } M \in M_{n}(\mathbf{k}) .
$$

(b) In order to distinguish these two $\epsilon$-unitary bialgebras of weight zero on $M_{n}(\mathbf{k})$, we call the $\epsilon$-unitary bialgebra of weight zero on $M_{n}(\mathbf{k})$ with the coproduct defined by Eq. (5) a Newtonian comatrix coalgebra.

(c) We would like to emphasize that there will be a classification of $\epsilon$-unitary bialgebraic structures (weight zero) on matrix algebras. Since $\epsilon$-unitary bialgebras of weight zero is closely related to associative Yang-Baxter equations, such a classification problem is related to the classification of solutions of the associative Yang-Baxter equation on matrix algebras.

Example 3.6. Consider the matrix algebra $M_{2}(\mathbf{k})$. By the definition of $\Delta_{\epsilon}$ in Eq. (5), we have

$$
\Delta_{\epsilon}\left[\begin{array}{ll}
1 & 0 \\
1 & 0
\end{array}\right]=\Delta_{\epsilon}\left[\begin{array}{ll}
1 & 0 \\
0 & 0
\end{array}\right]+\Delta_{\epsilon}\left[\begin{array}{ll}
0 & 0 \\
1 & 0
\end{array}\right]=-\left[\begin{array}{ll}
0 & 0 \\
1 & 0
\end{array}\right] \otimes\left[\begin{array}{ll}
0 & 0 \\
1 & 0
\end{array}\right]
$$

and

$$
\Delta_{\epsilon}\left[\begin{array}{ll}
0 & 1 \\
0 & 1
\end{array}\right]=\Delta_{\epsilon}\left[\begin{array}{ll}
0 & 1 \\
0 & 0
\end{array}\right]+\Delta_{\epsilon}\left[\begin{array}{ll}
0 & 0 \\
0 & 1
\end{array}\right]=\left[\begin{array}{ll}
1 & 0 \\
0 & 0
\end{array}\right] \otimes\left[\begin{array}{ll}
0 & 0 \\
0 & 1
\end{array}\right] \text {. }
$$

A directly calculation shows that

$$
\left(\Delta_{\epsilon} \otimes \mathrm{id}\right) \Delta_{\epsilon}\left[\begin{array}{ll}
1 & 0 \\
1 & 0
\end{array}\right]=\left(\mathrm{id} \otimes \Delta_{\epsilon}\right) \Delta_{\epsilon}\left[\begin{array}{ll}
1 & 0 \\
1 & 0
\end{array}\right]=\left[\begin{array}{ll}
0 & 0 \\
1 & 0
\end{array}\right] \otimes\left[\begin{array}{ll}
0 & 0 \\
1 & 0
\end{array}\right] \otimes\left[\begin{array}{ll}
0 & 0 \\
1 & 0
\end{array}\right]
$$

and

$$
\left(\Delta_{\epsilon} \otimes \mathrm{id}\right) \Delta_{\epsilon}\left[\begin{array}{ll}
0 & 1 \\
0 & 1
\end{array}\right]=\left(\mathrm{id} \otimes \Delta_{\epsilon}\right) \Delta_{\epsilon}\left[\begin{array}{ll}
0 & 1 \\
0 & 1
\end{array}\right]=0 \text {. }
$$


Moreover

$$
\begin{aligned}
\Delta_{\epsilon}\left(\left[\begin{array}{ll}
1 & 0 \\
1 & 0
\end{array}\right]\right) \cdot\left[\begin{array}{ll}
0 & 1 \\
0 & 1
\end{array}\right]+\left[\begin{array}{ll}
1 & 0 \\
1 & 0
\end{array}\right] \cdot \Delta_{\epsilon}\left(\left[\begin{array}{ll}
0 & 1 \\
0 & 1
\end{array}\right]\right) & =-\left[\begin{array}{ll}
0 & 0 \\
1 & 0
\end{array}\right] \otimes\left[\begin{array}{ll}
0 & 0 \\
0 & 1
\end{array}\right]+\left[\begin{array}{ll}
1 & 0 \\
1 & 0
\end{array}\right] \otimes\left[\begin{array}{ll}
0 & 0 \\
0 & 1
\end{array}\right] \\
& =\left[\begin{array}{ll}
1 & 0 \\
0 & 0
\end{array}\right] \otimes\left[\begin{array}{ll}
0 & 0 \\
0 & 1
\end{array}\right]=\Delta_{\epsilon}\left[\begin{array}{ll}
0 & 1 \\
0 & 1
\end{array}\right] \\
& =\Delta_{\epsilon}\left(\left[\begin{array}{ll}
1 & 0 \\
1 & 0
\end{array}\right] \cdot\left[\begin{array}{ll}
0 & 1 \\
0 & 1
\end{array}\right]\right) .
\end{aligned}
$$

3.2. An infinitesimal unitary Hopf algebra on matrix algebras. In this subsection, we equip the $\epsilon$-unitary bialgebra $\left(M_{n}(\mathbf{k}), \mathfrak{m}, E, \Delta_{\epsilon}\right)$ of weight zero with an antipode such that it is further an $\epsilon$-unitary Hopf algebra, under the view of Aguiar [1].

Lemma 3.7. Let $\left(M_{n}(\mathbf{k}), \mathfrak{m}, E, \Delta_{\epsilon}\right)$ be the $\epsilon$-unitary bialgebra of weight zero in Theorem 3.4 and

$$
D:=m \Delta_{\epsilon}: M_{n}(\mathbf{k}) \rightarrow M_{n}(\mathbf{k}) .
$$

Then for each $k \geq 0$ and $M \in M_{n}(\mathbf{k}), D^{*(k+1)}\left(M_{n}(\mathbf{k})\right)=0$ and so $D$ is locally nilpotent.

Proof. It suffices to prove the first statement by induction on $k \geq 0$. Using Sweedler notation, we may write

$$
\Delta_{\epsilon}(M)=\sum_{(M)} M_{(1)} \otimes M_{(2)} \text { for } M \in M_{n}(\mathbf{k})
$$

For the initial step of $k=0$, it follows from Eq. (5) that

$$
D^{*(1)}(M)=D^{*(1)}(M)=\sum_{(M)} D\left(M_{(1)}\right) D\left(M_{(2)}\right)=0,
$$

where the last step employs

$$
D\left(E_{i j}\right)=m \Delta_{\epsilon}\left(E_{i j}\right)= \begin{cases}\sum_{s=i}^{j-1} E_{i s} E_{(s+1) j}=0 & \text { if } i<j, \\ 0 & \text { if } i=j, \\ -\sum_{s=j}^{i-1} E_{i s} E_{(s+1) j}=0 & \text { if } i>j .\end{cases}
$$

Assume the result is true for $k=\ell$ for an $\ell \geq 1$, and consider the case when $k=\ell+1$. Then

$$
D^{*(\ell+1)}(M)=\left(D^{* \ell} * D\right)(M)=\mathfrak{m}\left(D^{* \ell} \otimes D\right) \Delta_{\epsilon}(M)=\sum_{(M)} D^{* \ell}\left(M_{(1)}\right) D\left(M_{(2)}\right)=0 .
$$

This completes the proof.

Theorem 3.8. Let $\mathbb{Q} \subseteq \mathbf{k}$. Then the quadruple $\left(M_{n}(\mathbf{k}), \mathrm{m}, E, \Delta_{\epsilon}\right)$ is an $\epsilon$-unitary Hopf algebra with the bijective antipode $S=-\sum_{n=0}^{\infty} \frac{1}{n !}(-D)^{n}$.

Proof. By Theorem 3.4, $\left(M_{n}(\mathbf{k}), \mathfrak{m}, E, \Delta_{\epsilon}\right)$ is an $\epsilon$-unitary bialgebra of weight zero. From Lemmas 2.11 and $3.7,\left(M_{n}(\mathbf{k}), \mathrm{m}, \Delta_{\epsilon}\right)$ is an $\epsilon$-Hopf algebra with bijective antipode $S=-\sum_{n=0}^{\infty} \frac{1}{n !}(-D)^{n}$. Then the result follows from Definition 2.8. 
3.3. A pre-Lie and a new Lie algebraic structures on matrix algebras. In this subsection, we first recall the concept of pre-Lie algebras and the connection between $\epsilon$-unitary bialgebras of weight zero and pre-Lie algebras. We then give a pre-Lie algebraic structure on a matrix algebra. Consequently, a new Lie algebraic structure on a matrix algebra is induced.

Definition 3.9. [33] A (left) pre-Lie algebra is a k-module $A$ together with a binary linear operation $\triangleright: A \otimes A \rightarrow A$ satisfying

$$
(a \triangleright b) \triangleright c-a \triangleright(b \triangleright c)=(b \triangleright a) \triangleright c-b \triangleright(a \triangleright c) \text { for } a, b, c \in A \text {. }
$$

Example 3.10. Here are two well-known pre-Lie algebras on dendriform dialgebras and RotaBaxter algebras, respectively.

(a) Let $(A, \prec,>)$ be a dendriform dialgebra. Then the multiplication $\star$ defined by $a \star b=a \prec$ $b+a>b$ gives an associative algebra. In addition, define

$$
\triangleright: A \otimes A \rightarrow A, a \otimes b \mapsto a>b-a<b \text { for } a, b \in A
$$

Then $A$ together with $\triangleright$ is a pre-Lie algebra [5].

(b) Let $(A, P)$ be a Rota-Baxter algebra of weight $\lambda$. If the weight $\lambda=0$, then the binary operation

$$
\triangleright: A \otimes A \rightarrow A, a \otimes b \mapsto P(a) \cdot b-b \cdot P(a) \text { for } a, b \in A,
$$

defines a pre-Lie algebra. If the weight $\lambda=-1$, then the binary operation

$$
\triangleright: A \otimes A \rightarrow A, a \otimes b \mapsto P(a) \cdot b-b \cdot P(a)-x \cdot y \text { for } a, b \in A,
$$

defines a pre-Lie algebra [6].

Let $(A, \triangleright)$ be a pre-Lie algebra. For any $a \in A$, let

$$
L_{a}: A \rightarrow A, \quad b \mapsto a \triangleright b
$$

be the left multiplication operator. Let

$$
L: A \rightarrow \operatorname{Hom}_{\mathbf{k}}(A, A), \quad a \mapsto L_{a} .
$$

The close relation between pre-Lie algebras and Lie algebras is characterized by the following two fundamental properties.

Lemma 3.11. (a) $[24$, Theorem 1] Let $(A, \triangleright)$ be a pre-Lie algebra. Define for elements in $A$ a new multiplication by setting

$$
[a, b]:=a \triangleright b-b \triangleright a \text { for } a, b \in A .
$$

Then $\left(A,\left[_{-},-\right]\right)$is a Lie algebra.

(b) $[8$, Proposition 1.2] Eq. (7) rewrites as

$$
L_{[a, b]}=L_{a} \circ L_{b}-L_{b} \circ L_{a}=\left[L_{a}, L_{b}\right],
$$

which implies that $L:\left(A,\left[_{-},,_{-}\right]\right) \rightarrow \operatorname{Hom}_{\mathbf{k}}(A, A)$ with $a \mapsto L_{a}$ gives a representation of the Lie algebra $\left(A,\left[_{-},{ }_{-}\right]\right)$.

Remark 3.12. By Lemma 3.11, a pre-Lie algebra induces a Lie algebra whose left multiplication operators give a representation of the associated commutator Lie algebra. 
Let $(A, m, 1, \Delta)$ be an $\epsilon$-unitary bialgebra of weight zero. Define

$$
\triangleright: A \otimes A \rightarrow A, a \otimes b \mapsto a \triangleright b:=\sum_{(b)} b_{(1)} a b_{(2)},
$$

where $b_{(1)}$ and $b_{(2)}$ are from the Sweedler notation $\Delta(b)=\sum_{(b)} b_{(1)} \otimes b_{(2)}$. The following result captures the connection from $\epsilon$-unitary bialgebras of weigh zero to pre-Lie algebras [5].

Lemma 3.13. [5] Let $(A, m, 1, \Delta)$ be an $\epsilon$-unitary bialgebra of weight zero. Then $A$ equipped with the $\triangleright$ in Eq. (9) is a pre-Lie algebra.

Remark 3.14. By Aguiar's construction about the pre-Lie product, a pre-Lie algebra from a weighted infinitesimal unitary bialgebra was derived in [23].

We now give a pre-Lie algebraic structure on matrix algebra $M_{n}(\mathbf{k})$. By linearity, we only need to define $E_{i j} \triangleright_{\varepsilon} E_{k l}$ for basis elements $E_{i j}, E_{k l} \in M_{n}(\mathbf{k})$. By Theorem 3.4, $\left(M_{n}(\mathbf{k}), m, E, \Delta_{\epsilon}\right)$ is an $\epsilon$-unitary bialgebra of weight zero. Applying Theorem 3.13 and Eq. (9), we define

$$
\triangleright_{\epsilon}: M_{n}(\mathbf{k}) \otimes M_{n}(\mathbf{k}) \rightarrow M_{n}(\mathbf{k}), \quad E_{i j} \triangleright_{\epsilon} E_{k l}:=\sum_{\left(E_{k l}\right)} E_{k l(1)} E_{i j} E_{k l(2)},
$$

where $E_{k l(1)}$ and $E_{k l(2)}$ are from $\Delta_{\epsilon}\left(E_{k l}\right)=\sum_{\left(E_{k l}\right)} E_{k l(1)} \otimes E_{k l(1)}$.

Theorem 3.15. The pair $\left(M_{n}(\mathbf{k}), \triangleright_{\epsilon}\right)$ is a pre-Lie algebra and so $\left(M_{n}(\mathbf{k}),\left[{ }_{-},{ }_{-}\right]_{\epsilon}\right)$ is a Lie algebra, where the Lie bracket given by

$$
\left[E_{i j}, E_{k l}\right]_{\epsilon}= \begin{cases}\operatorname{sgn}(l-k) E_{k l} & \text { if } j=i+1, l \neq k+1,(i-k+0.5)(i-l+0.5)<0 \\ \operatorname{sgn}(i-j) E_{i j} & \text { if } j \neq i+1, l=k+1,(k-i+0.5)(k-j+0.5)<0 \\ 0 & \text { otherwise. }\end{cases}
$$

Proof. By Theorem 3.4 and Lemma 3.13, $\left(M_{n}(\mathbf{k}), \triangleright_{\epsilon}\right)$ is a pre-Lie algebra. The remainder follows from Lemma 3.11 (a). Moreover, by Eqs. (5) and (10), we have

$$
E_{i j} \triangleright_{\epsilon} E_{k l}=\left\{\begin{array}{ll}
E_{k l} & \text { if } k<j=i+1 \leq l, \\
-E_{k l} & \text { if } l<j=i+1 \leq k, \\
0 & \text { otherwise }
\end{array} \quad E_{k l} \triangleright_{\epsilon} E_{i j}= \begin{cases}E_{i j} & \text { if } i<l=k+1 \leq j, \\
-E_{i j} & \text { if } j<l=k+1 \leq i, \\
0 & \text { otherwise }\end{cases}\right.
$$

Applying Eq. (8) in Lemma 3.11 (a), we obtain

$$
\left[E_{i j}, E_{k l}\right]_{\epsilon}= \begin{cases}E_{k l}-E_{i j} & \text { if } j=i+1=l=k+1, \\ E_{k l} & \text { if } k<j=i+1 \leq l, l \neq k+1, \\ -E_{k l} & \text { if } l<j=i+1 \leq k, \\ -E_{i j} & \text { if } i<l=k+1 \leq j, j \neq i+1, \\ E_{i j} & \text { if } j<l=k+1 \leq i, \\ 0 & \text { otherwise. }\end{cases}
$$

Then Eq. (11) follows by summing up Eq. (12).

Remark 3.16. (a) We emphasize that our Lie bracket $\left[{ }_{-},{ }_{-}\right]_{\epsilon}$ is different from the classical Lie bracket $\left[E_{i j}, E_{k l}\right]=\delta_{j k} E_{i l}-\delta_{l i} E_{k j}$ on matrix algebras.

(b) We call the Lie bracket $\left[{ }_{-},{ }_{-}\right]_{\epsilon}$ an $\epsilon$-Lie bracket which is induced by a weighted $\epsilon$-(unitary) bialgebraic structure. 
WEIGHTED INFINITESIMAL UNITARY BIALGEBRAS, MATRIX ALGEBRAS AND POLYNOMIAL ALGEBRAS 13

Example 3.17. Consider the matrix algebra $M_{2}(\mathbf{k})$. Let

$$
M=\left[\begin{array}{ll}
1 & 0 \\
1 & 0
\end{array}\right], \quad N=\left[\begin{array}{ll}
0 & 1 \\
0 & 1
\end{array}\right] .
$$

Then $M=E_{11}+E_{21}$ and $N=E_{12}+E_{22}$. By Theorem 3.15, we have

$$
\begin{aligned}
{[M, N]_{\epsilon}=\left[E_{11}+E_{21}, E_{12}+E_{22}\right]_{\epsilon} } & =\left[E_{11}, E_{12}\right]_{\epsilon}+\left[E_{11}, E_{22}\right]_{\epsilon}+\left[E_{21}, E_{12}\right]_{\epsilon}+\left[E_{21}, E_{22}\right]_{\epsilon} \\
& =\left[E_{21}, E_{12}\right]_{\epsilon}=E_{21} .
\end{aligned}
$$

By Example 3.6 and Lemma 3.13, we also have

$$
\begin{aligned}
{[M, N]_{\epsilon}=\sum_{N} N_{(1)} M N_{(2)}-\sum_{M} M_{(1)} N M_{(2)} } & =\left[\begin{array}{ll}
1 & 0 \\
0 & 0
\end{array}\right]\left[\begin{array}{ll}
1 & 0 \\
1 & 0
\end{array}\right]\left[\begin{array}{ll}
0 & 0 \\
0 & 1
\end{array}\right]+\left[\begin{array}{ll}
0 & 0 \\
1 & 0
\end{array}\right]\left[\begin{array}{ll}
0 & 1 \\
0 & 1
\end{array}\right]\left[\begin{array}{ll}
0 & 0 \\
1 & 0
\end{array}\right] \\
& =\left[\begin{array}{ll}
0 & 0 \\
1 & 0
\end{array}\right]=E_{21} .
\end{aligned}
$$

\section{WEIGHTED INFINITESIMAL UNITARY BIALGEBRAS ON POLYNOMIAL ALGEBRAS}

In this section, we derive a weighted $\epsilon$-unitary bialgebraic structure from a non-commutative polynomial algebra.

Definition 4.1. Let $\mathbf{k}$ be a unitary commutative ring. A non-commutative polynomial algebra $\mathbf{k}\left\langle x_{1}, \ldots, x_{n}\right\rangle$ with coefficients in $\mathbf{k}$ is a free algebra generated by $\left\{x_{1}, \ldots, x_{n}\right\}$.

Denote by

$$
\text { Mon }:=\left\{x_{i_{1}}^{\alpha_{1}} x_{i_{2}}^{\alpha_{2}} \cdots x_{i_{m}}^{\alpha_{m}} \mid 1 \leq i_{1}, i_{2}, \ldots, i_{m} \leq n, \alpha_{k} \in \mathbb{N}\right\} .
$$

Then the elements in Mon are called monomials in $\mathbf{k}\left\langle x_{1}, \ldots, x_{n}\right\rangle$ which are the elements from the set of all words in $\left\{x_{1}, \ldots, x_{n}\right\}$. Note that Mon is a $\mathbf{k}$-basis of $\mathbf{k}\left\langle x_{1} \ldots, x_{n}\right\rangle$ and Mon is a free monoid with the identity $x_{0}:=1$. We denote the multiplication on $\mathbf{k}\left\langle x_{1}, \ldots, x_{n}\right\rangle$ by $m$.

For any word $w \in$ Mon with length $l(w)=n$, we define a new notation to choose some elements of $w$. Denote by

$w[i, j]:=$ the $i$-th element to the $j$-th element of $w$ if $1 \leq i \leq j \leq n$.

Example 4.2. Consider the polynomial algebra $\mathbf{k}\langle x, y\rangle$. Let $w=x x y x y$. Then

$$
w[1,4]=x x y x, w[3,3]=y \text { and } w[2,3]=x y .
$$

Let us now define a coproduct on $\mathbf{k}\left\langle x_{1}, \ldots, x_{n}\right\rangle$ such that it is further an $\epsilon$-unitary bialgebra of weight $\lambda$. For any word $w \in$ Mon, define

$$
\Delta_{\epsilon}(w):= \begin{cases}0 & \text { if } w=0, \\ -\lambda(1 \otimes 1) & \text { if } w=1, \\ \sum_{i=1}^{n} w[1, i] \otimes w[i, n]+\lambda \sum_{i=1}^{n-1} w[1, i] \otimes w[i+1, n] & \text { if } l(w)=n>0 .\end{cases}
$$

We observe that $\mathbf{k}\left\langle x_{1}, \ldots, x_{n}\right\rangle$ is closed under the coproduct $\Delta_{\epsilon}$.

Lemma 4.3. Let $w_{1}, w_{2} \in \mathbf{k}\left\langle x_{1}, \ldots, x_{n}\right\rangle$. Then

$$
\Delta_{\epsilon}\left(w_{1} w_{2}\right)=w_{1} \cdot \Delta_{\epsilon}\left(w_{2}\right)+\Delta_{\epsilon}\left(w_{1}\right) \cdot w_{2}+\lambda w_{1} \otimes w_{2} .
$$


Proof. It suffices to consider basis elements $w_{1}, w_{2} \in$ Mon by linearity. Without loss of generality, we may suppose that $l\left(w_{1}\right)=m \geq 0$ and $l\left(w_{2}\right)=n \geq 0$ and so $w_{1} w_{2}=w$ is a new word of length $m+n$. If $w_{i}=0$ or 1 , then we have done. Consider $m \geq 1$ and $n \geq 1$. By Eq. (14), we have

$$
\begin{aligned}
& w_{1} \cdot \Delta_{\epsilon}\left(w_{2}\right)+\Delta_{\epsilon}\left(w_{1}\right) \cdot w_{2}+\lambda w_{1} \otimes w_{2} \\
= & w_{1} \cdot\left(\sum_{i=1}^{n} w_{2}[1, i] \otimes w_{2}[i, n]+\lambda \sum_{i=1}^{n-1} w_{2}[1, i] \otimes w_{2}[i+1, n]\right) \\
& +\left(\sum_{i=1}^{m} w_{1}[1, i] \otimes w_{1}[i, m]+\lambda \sum_{i=1}^{m-1} w_{1}[1, i] \otimes w_{1}[i+1, m]\right) \cdot w_{2}+\lambda w_{1} \otimes w_{2} \quad \text { (by Eq. (14)) } \\
= & \sum_{i=m+1}^{m+n} w[1, i] \otimes w[i, m+n]+\lambda \sum_{i=m+1}^{m+n-1} w[1, i] \otimes w[i+1, m+n] \\
& +\sum_{i=1}^{m} w[1, i] \otimes w[i, m+n]+\lambda \sum_{i=1}^{m-1} w[1, i] \otimes w[i+1, m+n]+\lambda w_{1} \otimes w_{2} \\
= & \sum_{i=m+1}^{m+n} w[1, i] \otimes w[i, m+n]+\lambda \sum_{i=m+1}^{m+n-1} w[1, i] \otimes w[i+1, m+n] \\
& +\sum_{i=1}^{m} w[1, i] \otimes w[i, m+n]+\lambda \sum_{i=1}^{m-1} w[1, i] \otimes w[i+1, m+n]+\lambda w[1, m] \otimes w[m+1, m+n] \\
= & \sum_{i=1}^{m+n} w[1, i] \otimes w[i, m+n]+\lambda \sum_{i=1}^{m+n-1} w[1, i] \otimes w[i+1, m+n] \\
= & \Delta_{\epsilon}(w)=\Delta_{\epsilon}\left(w_{1} w w_{2}\right) \quad(\text { by Eq. }(14),
\end{aligned}
$$

as desired.

Lemma 4.4. The pair $\left(\mathbf{k}\left\langle x_{1}, \ldots, x_{n}\right\rangle, \Delta_{\epsilon}\right)$ is a coalgebra (without counit).

Proof. It is enough to check the coassociative law:

$$
\left(\Delta_{\epsilon} \otimes \mathrm{id}\right) \Delta_{\epsilon}(w)=\left(\mathrm{id} \otimes \Delta_{\epsilon}\right) \Delta_{\epsilon}(w) \text { for } w \in \text { Mon. }
$$

When $w$ is 0 or 1 , then the result holds for trivially. Consider $l(w) \geq 1$. On the one hand,

$$
\begin{aligned}
& \left(\Delta_{\epsilon} \otimes \mathrm{id}\right) \Delta_{\epsilon}(w) \\
= & \left(\Delta_{\epsilon} \otimes \mathrm{id}\right)\left(\sum_{i=1}^{n} w[1, i] \otimes w[i, n]+\lambda \sum_{i=1}^{n-1} w[1, i] \otimes w[i+1, n]\right) \quad \text { (by Eq. (14)) } \\
= & \sum_{i=1}^{n} \Delta_{\epsilon}(w[1, i]) \otimes w[i, n]+\lambda \sum_{i=1}^{n-1} \Delta_{\epsilon}(w[1, i]) \otimes w[i+1, n] \\
= & \sum_{i=1}^{n}\left(\sum_{j=1}^{i} w[1, j] \otimes w[j, i]+\lambda \sum_{j=1}^{i-1} w[1, j] \otimes w[j+1, i]\right) \otimes w[i, n] \\
& +\lambda \sum_{i=1}^{n-1}\left(\sum_{j=1}^{i} w[1, j] \otimes w[j, i]+\lambda \sum_{j=1}^{i-1} w[1, j] \otimes w[j+1, i]\right) \otimes w[i+1, n] \quad \text { (by Eq. (14)) }
\end{aligned}
$$


WEIGHTED INFINITESIMAL UNITARY BIALGEBRAS, MATRIX ALGEBRAS AND POLYNOMIAL ALGEBRAS 15

$$
\begin{aligned}
= & \left.\sum_{i=1}^{n} \sum_{j=1}^{i} w[1, j] \otimes w[j, i] \otimes w[i, n]+\lambda \sum_{i=1}^{n} \sum_{j=1}^{i-1} w[1, j] \otimes w[j+1, i] \otimes w[i, n]\right) \\
& +\lambda \sum_{i=1}^{n-1} \sum_{j=1}^{i} w[1, j] \otimes w[j, i] \otimes w[i+1, n]+\lambda^{2} \sum_{i=1}^{n-1} \sum_{j=1}^{i-1} w[1, j] \otimes w[j+1, i] \otimes w[i+1, n] \\
= & \left.\sum_{i=1}^{n} \sum_{j=1}^{i} w[1, j] \otimes w[j, i] \otimes w[i, n]+\lambda \sum_{i=2}^{n} \sum_{j=1}^{i-1} w[1, j] \otimes w[j+1, i] \otimes w[i, n]\right) \\
& +\lambda \sum_{i=1}^{n-1} \sum_{j=1}^{i} w[1, j] \otimes w[j, i] \otimes w[i+1, n]+\lambda^{2} \sum_{i=2}^{n-1} \sum_{j=1}^{i-1} w[1, j] \otimes w[j+1, i] \otimes w[i+1, n] .
\end{aligned}
$$

On the other hand,

$$
\begin{aligned}
& \left(\mathrm{id} \otimes \Delta_{\epsilon}\right) \Delta_{\epsilon}(w) \\
= & \left(\mathrm{id} \otimes \Delta_{\epsilon}\right)\left(\sum_{i=1}^{n} w[1, i] \otimes w[i, n]+\lambda \sum_{i=1}^{n-1} w[1, i] \otimes w[i+1, n]\right) \quad \text { (by Eq. (14)) } \\
= & \sum_{i=1}^{n} w[1, i] \otimes \Delta_{\epsilon}(w[i, n])+\lambda \sum_{i=1}^{n-1} w[1, i] \otimes \Delta_{\epsilon}(w[i+1, n]) \\
= & \sum_{i=1}^{n} w[1, i] \otimes\left(\sum_{j=i}^{n} w[i, j] \otimes w[j, n]+\lambda \sum_{j=i}^{n-1} w[i, j] \otimes w[j+1, n]\right) \\
& \left.+\lambda \sum_{i=1}^{n-1} w[1, i] \otimes\left(\sum_{j=i+1}^{n} w[i+1, j] \otimes w[j, n]+\lambda \sum_{j=i+1}^{n-1} w[i+1, j] \otimes w[j+1, n]\right) \quad \text { (by Eq. (1) }\right) \\
= & \sum_{i=1}^{n} \sum_{j=i}^{n} w[1, i] \otimes w[i, j] \otimes w[j, n]+\lambda \sum_{i=1}^{n} \sum_{j=i}^{n-1} w[1, i] \otimes w[i, j] \otimes w[j+1, n] \\
& +\lambda \sum_{i=1}^{n-1} \sum_{j=i+1}^{n} w[1, i] \otimes w[i+1, j] \otimes w[j, n]+\lambda^{2} \sum_{i=1}^{n-1} \sum_{j=i+1}^{n-1} w[1, i] \otimes w[i+1, j] \otimes w[j+1, n] \\
& +\lambda \sum_{j=2}^{n} \sum_{i=1}^{n} \sum_{i=1}^{n} \sum_{j=i} w[1, i] \otimes w[i, j] \otimes w[j, n]+\lambda \sum_{i=1}^{n-1} \sum_{j=i}^{n-1} w[1, i] \otimes w[i, j] \otimes w[j+1, n] \\
& +\lambda \sum_{i=1}^{n-1} \sum_{j=i+1}^{n} w[1, i] \otimes w[i+1, j] \otimes w[j, n]+\lambda^{2} \sum_{i=1}^{n-2} \sum_{j=i+1}^{n-1} w[1, i] \otimes w[i+1, j] \otimes w[j+1, n] \\
= & \sum_{j}^{n} \sum_{j=1}^{n} w[1, i] \otimes w[i, j] \otimes w[j, n]+\lambda \sum_{j=1}^{n-1} \sum_{i=1}^{j} w[1, i] \otimes w[i, j] \otimes w[j+1, n] \\
&
\end{aligned}
$$

(by exchanging the order of $i$ and $j$ in all sums) 


$$
\begin{aligned}
= & \sum_{i=1}^{n} \sum_{j=1}^{i} w[1, j] \otimes w[j, i] \otimes w[i, n]+\lambda \sum_{i=1}^{n-1} \sum_{j=1}^{i} w[1, j] \otimes w[j, i] \otimes w[i+1, n] \\
& +\lambda \sum_{i=2}^{n} \sum_{j=1}^{i-1} w[1, j] \otimes w[j+1, i] \otimes w[i, n]+\lambda^{2} \sum_{i=2}^{n-1} \sum_{j=1}^{i-1} w[1, j] \otimes w[j+1, i] \otimes w[i+1, n]
\end{aligned}
$$

(by exchanging the index of $i$ and $j$ ).

This completes the proof.

Now we state our main result in this section.

Theorem 4.5. The quadruple $\left(\mathbf{k}\left\langle x_{1}, \ldots, x_{n}\right\rangle, m, 1, \Delta_{\epsilon}\right)$ is an $\epsilon$-unitary bialgebra of weight $\lambda$.

Proof. It follows from the Lemmas 4.3 and 4.4.

Example 4.6. Consider the polynomial algebra $\mathbf{k}\langle x, y\rangle$. Let $w_{1}=x y, w_{2}=y x y$ be two words in $\mathbf{k}\langle x, y\rangle$. By the definition of $\Delta_{\epsilon}$ in Eq. (14), we have

$$
\Delta_{\epsilon}\left(w_{1}\right)=x y \otimes y+x \otimes x y+\lambda x \otimes y
$$

and

$$
\Delta_{\epsilon}\left(w_{2}\right)=y x y \otimes y+y x \otimes x y+y \otimes y x y+\lambda(y x \otimes y+y \otimes x y) .
$$

Then

$$
\begin{aligned}
& \left(\Delta_{\epsilon} \otimes \mathrm{id}\right) \Delta_{\epsilon}\left(w_{1}\right) \\
= & \left.\left(\Delta_{\epsilon} \otimes \mathrm{id}\right)(x y \otimes y+x \otimes x y+\lambda x \otimes y) \quad \text { (by Eq. (17) }\right) \\
= & (x y \otimes y+x \otimes x y+\lambda x \otimes y) \otimes y+x \otimes x \otimes x y+\lambda x \otimes x \otimes y \quad \text { (by Eq. (14) }) \\
= & x \otimes x y \otimes y+x y \otimes y \otimes y+x \otimes x \otimes x y+\lambda x \otimes y \otimes y+\lambda x \otimes x \otimes y \\
= & x \otimes(x y \otimes y+x \otimes x y+\lambda x \otimes y)+x y \otimes y \otimes y+\lambda x \otimes y \otimes y \\
= & x \otimes \Delta_{\epsilon}(x y)+x y \otimes \Delta_{\epsilon}(y)+\lambda x \otimes \Delta_{\epsilon}(y) \quad \text { (by Eq. (14)) } \\
= & \left(\mathrm{id} \otimes \Delta_{\epsilon}\right)(x \otimes x y+x y \otimes y+\lambda x \otimes y) \\
= & \left(\mathrm{id} \otimes \Delta_{\epsilon}\right) \Delta_{\epsilon}(x y) \quad \text { (by Eq. (17)). }
\end{aligned}
$$

Similarly,

$$
\begin{aligned}
& \left(\Delta_{\epsilon} \otimes \mathrm{id}\right) \Delta_{\epsilon}\left(w_{2}\right) \\
= & \left(\Delta_{\epsilon} \otimes \mathrm{id}\right)(y x y \otimes y+y x \otimes x y+y \otimes y x y+\lambda(y x \otimes y+y \otimes x y)) \quad \text { (by Eq. (18)) } \\
= & y \otimes(y x y \otimes y+y x \otimes x y+y \otimes y x y+\lambda(y x \otimes y+y \otimes x y))+y x \otimes(x y \otimes y+x \otimes x y+\lambda x \otimes y) \\
& +y x y \otimes y \otimes y+\lambda(y \otimes(x \otimes x y+x y \otimes y+\lambda x \otimes y))+\lambda y x \otimes y \otimes y \\
= & y \otimes \Delta_{\epsilon}(y x y)+y x \otimes \Delta_{\epsilon}(x y)+y x y \otimes \Delta_{\epsilon}(y)+\lambda y \otimes \Delta_{\epsilon}(x y)+\lambda y x \otimes \Delta_{\epsilon}(y) \quad \text { (by Eq. (14)) } \\
= & \left(\mathrm{id} \otimes \Delta_{\epsilon}\right)(y \otimes y x y+y x \otimes x y+y x y \otimes y+\lambda y \otimes x y+\lambda y x \otimes y) \\
= & \left(\mathrm{id} \otimes \Delta_{\epsilon}\right) \Delta_{\epsilon}\left(w_{2}\right) \quad \text { (by Eq. (18)). }
\end{aligned}
$$

A directly calculation shows that $w_{1} \cdot \Delta_{\epsilon}\left(w_{2}\right)+\Delta_{\epsilon}\left(w_{1}\right) \cdot w_{2}+\lambda w_{1} \otimes w_{2}=\Delta_{\epsilon}(x y) \cdot y x y+x y \cdot \Delta_{\epsilon}(y x y)+\lambda x y \otimes y x y$ $=x \otimes x y y x y+x y \otimes y y x y+x y y \otimes y x y+x y y x \otimes x y+x y y x y \otimes y$ 
WEIGHTED INFINITESIMAL UNITARY BIALGEBRAS, MATRIX ALGEBRAS AND POLYNOMIAL ALGEBRAS 17

$$
\begin{aligned}
& +\lambda(x \otimes y y x y+x y \otimes y x y+x y y \otimes x y+x y y x \otimes y) \\
& =\Delta_{\epsilon}(x y y x y)=\Delta_{\epsilon}\left(w_{1} w_{2}\right) \quad(\text { by Eq. }(14)) .
\end{aligned}
$$

Acknowledgments: We thank the anonymous referee for valuable suggestions helping to improve the paper.

Funds: This work was supported by the National Natural Science Foundation of China (Grant No. 11771191).

\section{REFERENCES}

[1] M. Aguiar, Infinitesimal Hopf algebras, Contemporary Mathematics, New trends in Hopf algebra theory (La Falda, 1999), 1-29, Contemp. Math., 267, Amer. Math. Soc., Providence, RI, (2000). 2, 4, 5, 6, 10

[2] M. Aguiar, Pre-Poisson algebras, Lett. Math. Phys. 54 (2000), 263-277. 3

[3] M. Aguiar, On the associative analog of Lie bialgebras, J. Algebra 244 (2001), 492-532. 2

[4] M. Aguiar, Infinitesimal Hopf algebras and the cd-index of polytopes. Geometric combinatorics (San Francisco, CA/Davis, CA, 2000), Discrete Comput. Geom. 27 (2002), 3-28. 2

[5] M. Aguiar, Infinitesimal bialgebras, pre-Lie and dendriform algebras. Hopf algebras, 1-33, Lecture Notes in Pure and Appl. Math. 237, Dekker, New York, 2004. 2, 3, 6, 11, 12

[6] H. H. An and C. M. Bai, From Rota-Baxter algebras to pre-Lie algebras, J. Phys. A: Math. Theor. 41 (2008), 01520.3, 11

[7] A. Andrada and S. Salamon, Complex product structures on Lie algebras, Forum Math. 17 (2005), 261295.3

[8] C. M. Bai, Introduction to pre-Lie algebras, Preprint, available at http: // einspem. upm. edu. my/ equals8/ CSS/ pre-Lie.pdf 3, 11

[9] C. M. Bai, A unified algebraic approach to the classical Yang-Baxter equation, J. Phy. A: Math. Theor. 40 (2007), 11073-11082. 3

[10] C. M. Bai, L. Guo and X. Ni, O-operators on associative algebras and associative Yang-Baxter equations, Pacific J. Math. 256 (2012), 257-289. 3

[11] C. M. Bai, O. Bellier, L. Guo, and X. Ni, Splitting of operations, Manin products, and Rota-Baxter operators, Int. Math. Res. Not. IMRN (2013), 485-524. 3

[12] C. M. Bai, X. Gao, L. Guo and Y. Zhang, Weighted infinitesimal bialgebras and weighted infinitesimal Hopf modules, in preparation. 1

[13] M. Bordemann, Generalized Lax pairs, the modified classical Yang-Baxter equation, and affine geometry of Lie groups, Comm. Math. Phys. 135 (1990), 201-216. 3

[14] A. A. Balinskii and S. P. Novikov, Poisson brackets of hydrodynamic type, Frobenius algebras and Lie algebras, Dokl. Akad. Nauk SSSR 283 (1985), 1036-1039. 3

[15] A. Connes and D. Kreimer, Hopf algebras, renormalization and non-commutative geometry, Comm. Math. Phys. 199 (1998), 203-242. 3

[16] F. Chapoton and M. Livernet, Pre-Lie algebras and the rooted trees operad, Int. Math. Res. Not. IMRN 8 (2001), 395-408. 3

[17] V. Chari and A. Pressley, A guide to quantum groups, Cambridge University Press, Cambridge, 1994. 5

[18] R. Ehrenborg and M. Readdy, Coproducts and the cd-index, J. Algebraic Combin. (1998), 273-299. 2

[19] P. Etingof and A.Soloviev, Quantization of geometric classical $r$-matrices, Math. Res. Lett. 6 (1999), 223-228. 3

[20] L. Foissy, Quantization of the Hopf algebras of decorated planar rooted trees, preprint, 2008. 5

[21] L. Foissy, The infinitesimal Hopf algebra and the poset of planar forests, J. Algebraic Combin. 30 (2009), 277-309. 2

[22] L. Foissy, The infinitesimal Hopf algebra and the operads of planar forests, Int. Math. Res. Not. IMRN 3 (2010), 395-435. 2

[23] X. Gao and Y. Zhang, Planar rooted forests and weighted infinitesimal Hopf algebras: an operated algebra approach, arXiv:1810.10790. 1, 2, 3, 4, 12 
[24] M. Gerstenhaber, The cohomology structure of an associative ring, Ann. of Math. (2) 78 (1963), 267-288. 3,11

[25] L. Guo, An Introduction to Rota-Baxter Algebra, International Press, 2012. 3

[26] I. Z. Golubchik and V. V. Sokolov, Generalized operator Yang-Baxter equations, integrable ODEs and nonassociative algebras, J. Nonlinear Math. Phys. 7 (2000), 184-197. 3

[27] P. S. Hirschhorn and L. A. Raphael, Coalgebraic foundations of the method of divided differences. Adv. Math. 91 (1992), 75-135. 1, 5

[28] S. Joni and G.-C. Rota, Coalgebras and bialgebras in combinatorics, Stud. Appl. Math. 61 (1979), 93-139. $1,3,4$

[29] H. Kim, Complete left-invariant affine structures on nilpotent Lie groups, J. Differ. Geom. 24 (1986),37394. 3

[30] T. Y. Lam, Lectures on modules and rings. Graduate Texts in Mathematics, Springer-Verlag, New York, 1999. 7

[31] J.-L. Loday and M. O. Ronco, On the structure of cofree Hopf algebras, J. Reine Angew. Math. 592 (2006), 123-155. 2, 3, 4

[32] X. X. Li, D. P. Hou and C. M. Bai, Rota-Baxter operators on pre-lie algebras, J. Nonlinear Math. Phys. 14 (2007), 269-89 3

[33] D. Manchon, A short survey on pre-Lie algebras. Noncommutative geometry and physics: renormalisation, motives, index theory, 89-102, ESI Lect. Math. Phys., Eur. Math. Soc., Zürich, 2011. 11

[34] A. Medina, Flat left-invariant connections adapted to the automorphism structure of a Lie group, J. Diff. Geom. 16 (1981), 445-474. 3

[35] O. Ogievetsky and T. Popov, R-matrices in rime, Adv. Theor. Math. Phys. 14 (2010), 439-505. 1, 2

[36] J. Pei, C. M. Bai and L. Guo, Splitting of operads and Rota-Baxter operators on operads, Appl. Categ. Structures 25 (2017), 505-538. 3

[37] D. E. Radford, Hopf algebras, World Scientific Publishing Co. Pte. Ltd., Hackensack, NJ, 2012. 2

[38] E.B. Vinberg, The theory of homogeneous convex cones, Transl. Moscow Math. Soc. 2 (1963), 303-358. 3

[39] S. X. Wang and S. H. Wang, Drinfeld double for braided infinitesimal Hopf algebras, Comm. Algebra 42 (2014), 2195-2212.2

[40] Y. Zhang, D. Chen, X. Gao and Y. F. Luo, Weighted infinitesimal bialgebras, weihgted cocycles, and Pre-Lie algebras, to appear in Pacific J. Math. arXiv:1812.01452. 2

[41] Y. Zhang, X. Gao and Y. F. Luo, Weighted infinitesimal unitary bialgebras, pre-Lie and free monoid algebras, submitted. 5

[42] Y. Zhang, X. Gao and Y. F. Luo, Weighted infinitesimal unitary bialgebras and pre-Lie algebras on decorated rooted forests, submitted. 2

[43] Y. Zhang, X. Gao and J. W. Zheng, Weighted infinitesimal unitary bialgebras on matrix algebras and weighted associative Yang-Baxter equations, arXiv:1811.00842. 1, 2, 9

School of Mathematics and Statistics, Lanzhou University, Lanzhou, Gansu 730000, P. R. China

E-mail address: zhangy2016@1zu.edu.cn

School of Mathematics and Statistics, Lanzhou University, Lanzhou, Gansu 730000, P. R. China

E-mail address: zhengjw16@lzu.edu.cn

School of Mathematics and Statistics, Key Laboratory of Applied Mathematics and Complex Systems, Lanzhou University, LaNZhou, 730000, P. R. China

E-mail address: luoyf@lzu.edu.cn 